\title{
Actividad física en escolares chilenos normales y de talla baja
}

\author{
Vivien Gattás Z. ${ }^{\text {; }}$ Gladys Barrera A. ${ }^{2}$; José Riumalló S. ${ }^{3}$; Ricardo Uauy D. ${ }^{3}$ \\ Physical activity in normal and low height \\ for age chilean school children
}

\begin{abstract}
Physical acivily was evaluated n 70 male and female chilean scrool childre- oged 7 to 9 years. Verno cnd low height for cge childien were studied. Intomation from each individual was gatherad oy recall nterview and aclivity diany by the factarial methoc or tirne motion techrique. Dally lime distrikution sor cifferent physical activities was cbai-ed for a week day ard a weekerd. A trained observer recorded physical activities performed by each cinj along a 20 minulas breck in schocl duties. Mosi acivilies were of secentory hype. A striking finding wcs the time soent cn sdentary act vilies such as sleeping and lyne down $117.5 \pm 1.1$ to $12.0 \pm 1.4$ mours in normal and small for height boys and $11.7 \pm 1.21012 .2 \pm 1.6$ hcurs nn normel and small for meight gilst Both boys and girls spent an averoce 3 hours a doy watching TV on schocl doy and 4 hours ar inere o* weekends. No s gnificant differe-ces in lime distr bution of different prysical activities were found oetween romal and short stature child:en. Direct cbservotion shewed that normal boys spend significanlly la:ger proportion of their lime 129 . s\% in in moderate and heavy activi'ies

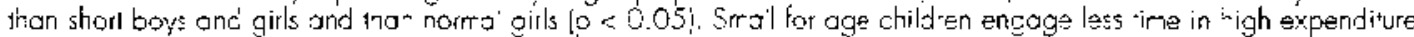
ccliv fies thon normal children. Thus, ir ceneral these chilean schoo: childen showed sedenlary life styles, with minimal physical activity anci they devcled $30 \%$ of perr daily lime to watch $T V$.
\end{abstract}

(Key words: activities of daiy living, exerc:se, ene'gy metabolism, expenditu'e, schoc' chi dren.f

En Chile se ha superado en los ültimos decenios la desnutrición aguda. Sin embargo, un número importante de escolares de primero y segundo año básico y condición socioeconómics baja, tienen talla baja ( 30 y $34 \%$ respectivamente), proporcion que aumenta progresivamente con la edad a $65 \%$ a la de 13 años'. Entre los problemas asociados con talla baja se mencionan menores actividad y capacidad de trabajo físic $0^{2}$.

Las prevalencias de sobrepeso y obesidad (según peso para là talla) en niños de 8 a 10 años de edad son, respectivamente. $17,8 \%$ y $9,3 \%$ en varones y 21,7 y $15,4 \%$ en mujeres ${ }^{3}$. La inactividad física precede a la obesidad en los jovenes y ésta a la del adulto y sus conse-

1. Nutracionista. Instituro de Nutricion y Tecnologín de Jos Alimentos (INTA). Liniversidad de Chile.

2. Enfermera Cniversitaria. Instituto de Nutrición y Tecnología de los Alimentos (INTA). Universidad de Chile.

3. Instituto de Nutrición y Tecnología de los Alimentos (INTA), Universidad de Chile. cuencias 4 . El ejercicio físico y el deporte practicados regularmente son importantes en la prevención de cufermedades crónicas frecuentes, como las cardiovasculares, muchas veces asociadas a sobrepeso $y$ obesidad ${ }^{5}, 6$. Existe poca información sobre patrones de actividad física entre los escolares y no se ha establecido el comportamiento de niños normales o los que sufren algún grado de desnutrición, como tampoco si las tallas más bajas sc asocian con efectos funcionales como, por ejemplo, limitación en la actividad física habitual cspontánea.

La promoción de su salud, requiere métodos prácticos para establecer el patrón de activida. des habituales, ocupacionales y de tiempo fibre en los escolares: en personas de otras edades se emplean cuestionarios de actividad física que permiten distinguir a las personas sedentarias de las más activas ${ }^{7} .8$.

En este estudio se compara la actividad física habitual en escolares de talla normal y baja y se la relaciona con el gasto cnergético diario y la condición mutricional. 


\section{Material y Método}

El estudio se Hevó a caho en 70 niños y niñas escolares de 7 a 9 años de edad, de baja condición socioeconómica. en dos escuelas trísicas de li connuna de Macul, en Santiago metropolitano. Se midieron sus peso y talla con mérodos estándar". El estado de nutrición se cyaluo de acuerdo a los patrones NCHS/OMS"', clasificando a los niños co. tho normales o de talla baja según las relaciones peso/talla (P/T) y tallafedad $(T / E)>1,0 \mathrm{DE} 0<-1 \mathrm{DE} \mathrm{DE}^{\prime \prime}$. La condiciốn socioeconómica se estimú con un protocole cstructurado basido en la escala de Graffar modificada ${ }^{12}$. confirmando el nivel socioeconómico bajo y la homoseneidar de la muestra mediante revisión de los tegistros de alumnos de cada cscuela. Mediante examen clínico realizado por un mético (anamnesis y exanen físico) se descartaron afecciones que por sí solas pudiesen modificar la actividad física. El gasto energético de reposo se midió con inćcodo de circuito abierto ${ }^{13}$. El consumo de $\mathrm{O}_{2}$ en airc espirado se midió despues de 30 min en condiciones de reposo mental y fisico, ambiente y temperatura confoitables y 12 horas despućs de la ólıima comida. El gasto calórico obtenido se utilizó como referencia para las horas de teposo.

El patron habitual de activiuad física se indago mediante entrevista estruclurada y observación directa de actividades recreativas escolares (método factorial o de tiempo en moviniento $)^{14}$. Las aclividades se agruparon de acuerdo a su intensidad, basándose en la clasificación FAO/OMSf UN' de $1985^{15}$, empleando un códjoo de registro para cada una de ellas. El patrón hnbitual de actividad física se estahleció mediante un protocolo lipo de entrevista estructurada por actividades habituales que realizaban los niños. La infonmación se obturo individualmente, para establecer el tiempo destinado por el niño a cada una de las actividades exijpuladas, más aquellas que pudiera éste señalar en forma específica. Se incluyeron las actividades de un día hábil y otro festivo desde el momento de levantarse en la mantans hasta la hora de acostarsc. estableciendo lapsos específicos que permitieron obtener informacióu detallada sabre ellas. Fl tiempo se expresó en horas. A fin de dar mayor precisión a los datos, en la entrevista estructurada realizada por un solo encuestador, la definición de duracjón de actividades se hizo tamando como puntos de referencin a hitos familiates, tales como horarios de conidas. programas de televisión, hora de entrada y salida del colegio y otros.

Además se cronometruon las actividades recrentivas o discrecionales de los niños por observación dirceta e individenl y por un período de 20 minutos, correspondiente al tiempo destinado a recreo en la escuela fmétodo factorial o de tientpo movimiento). Para ello se utilizó una cartilia de registro minutado. construida en hase a un número de casiJleros correspondiente al múunero de minutos previstos dc duración de la actividad global. Conjuntamente. se elaboró la nomenclatuta y codificación accesoria urilizada en el registro de actividades o transferencia de lo observado al correspondiente casillero a rinuto.

Parn eronometrar las actividades. el observador se ubicó en un punto que le permitiese ver todo el espacio de desplazamiento del niño. Para medir el tiempo de desplazamien1o en las actividades realizadas se usaron dos cronómetros simples: en el primero se registró la duración global del período obscrvado $y$, en el segundo. el tiempo de desplazamiento en un espacio determinado, empleado en las diferentes actividades parciales registradas por el nin̄o. Se efectuó utia agrupación de los diferentes tipos de artividades realizadas, se hizo un resumen de los tiempos parciales en casla actividad. se calcutó el valor promedio en ninutos del grupo según la clasificación.

Para calcular el gasto energético se tomó comno base el metabolismo de reposo, medido por calorimetría indirecta. A] gasto energético de metabolismo de reposo por hora se aplicaron los factores por catcgorías de actividad en reposo. ligeras, moderadas e intensas segŭn FAO/OMS/UNU $1985^{15}$. E] cálculo de gasio energético en 24 horas se determinó separidamente por días de semana y festivos.

La ingesta de alimentos se estimb mediante el mćtodv recordatorio de 24 boras ${ }^{\text {lh }}$, en Jíts no consecutivos y considerando días de semana y festivos.

Para el anctisis estadj́stico se calculó promedio y desviación estándar de cada una de las variables, Las diferercias de promedios entre pares de grupos de talla baja $y$ normales se estimaron mediante la prueba de " $e$ " te Student y para establecer la variabilidad de los 4 grupos se utilizó análisis Je varianza (ANOVA) ${ }^{17, ~} 1 \mathrm{.}$.

\section{Resultados}

La cvaluación antropométrica del cstado nutricional permitió caracterizar los grupos normales y de talla baja, cuyas relaciones P/T y T/ E fueron expresadas en puntaje Z. La edad promedio de los grupos estudiados fue de 8,7 aíros, peso corporal 27,8 y $27,5 \mathrm{~kg}$ para varones y niñas normales y de 22,9 y $25,3 \mathrm{~kg}$ en varones y niñas con déficit de taila, respectivamente. La talla fue de $129 \mathrm{~cm}$ para los grupos normales y Je $121 \mathrm{~cm}$ para los con déficit.

No se encontraron diferencias significativas en relación a los indicadores $P / T$ e indice de masa corporal (IMC), sin embargo, como cra de esperar, la T/E fue significativamente menor en los niños de uno y otro sexo con talla baja $(-4$ DE) con respecto a sus pares normales (tabla I).

Los niños y niñas normales y los de talla baja dormían alrededor de 12 horas diarias, durante los días de la semana y en los festivos, sin diferencias en las horas de sueño entre los cuatro grupos. El tiempo destinado a televisión. tanto en días de semana y festivos, ocupaba prícticamente un tercio de las horas de vigilia, siendo esta actividad significativamente mayor en días festivos, tanto entre cscolares normales como los de talla baja (tabla 2).

El análisis del tiempo destinado para cada actividad (tabla 3) y para éstas agrupadas según Ia clasificación señalada en el método, mostró que los varones normales y los de talla baja des- 
Tabla 1

Características antropométricas y estado nutricional de varones y mujeres

\begin{tabular}{|c|c|c|c|c|}
\hline & \multicolumn{2}{|c|}{ Yarones (n; 41) } & \multicolumn{2}{|c|}{ Mujeres (n: 29) } \\
\hline & $\begin{array}{l}\text { Normales } \\
\text { (d: } 23)\end{array}$ & $\begin{array}{c}\text { Talla haja } \\
\text { (n: 18) }\end{array}$ & $\begin{array}{c}\text { Normales } \\
(\mathrm{n}: 17)\end{array}$ & $\begin{array}{c}\text { Talla baja } \\
\text { (n: 11) }\end{array}$ \\
\hline Echad (ânus) & $8.7 \pm 0.7$ & $8.8 \pm 0,7$ & $8.6 \pm(1.6$ & $8.8 \pm 0.6$ \\
\hline Peso $(k \mathrm{~kg})$ & $27,8 \pm 2,8^{4}$ & $22.9 \pm 2.6$ & $27,5 \pm 3.9$ & $25,3 \pm 2.9$ \\
\hline Talla $(\mathrm{cm})$ & $129,1 \pm 4,8^{i}$ & $120.9 \pm 4.0$ & $129.2 \pm 5,4^{*}$ & $122.2 \pm 3.2$ \\
\hline $\mathrm{P} / \mathrm{T}(\mathrm{Z})^{* * *}$ & $-0.4 \pm 0,6$ & $0,01 \pm 0,7$ & $0.2 \pm 0.5$ & $0,8 \pm 0,6$ \\
\hline $\mathrm{T} / \mathrm{E}(\mathrm{Z})^{* *}$ & $-0,4 \pm 0,4$ & $-1.8 \pm 0.4$ & $-0,1 \pm 0,5$ & $-1,4 \pm 0,3$ \\
\hline $\mathrm{MMC}\left(\mathrm{kg} / \mathrm{m}^{2}\right)$ & $16,7 \pm 1,1$ & $15.6 \pm 1,1$ & $16.4 \pm 1.3$ & $16.9 \pm 1.4$ \\
\hline
\end{tabular}

* $p<0.000 \quad *$ *stíndar OMS P/T: pesoltalla $\quad$ T/E: rallipedad

IMC: Indice de masa corporal.

Tabla 2

Distribución del liempo (h) según tipo de actividad en yaroncs.

Día de semana y festivo $(\bar{x} \pm D E)$

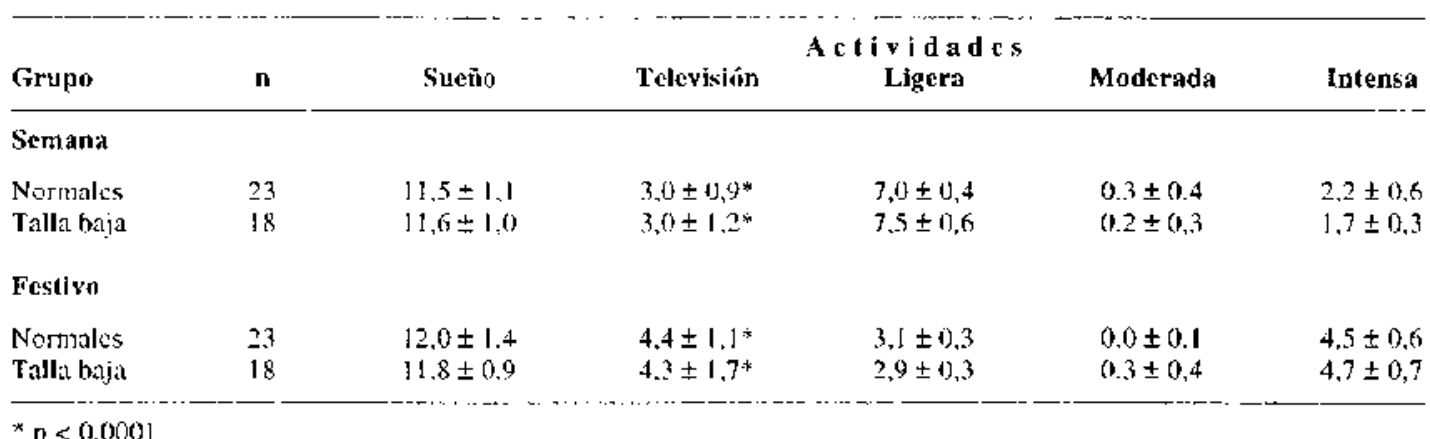

${ }^{*} \mathrm{p}<0.0001$

tinaron mayor nûmero de horas a actividades ligeras durante la semana. En días festivos, en cambio, aumentaron a un promedio de 3 horas diarias las actividades intensas. Una situación similar se observó en las niñas, que aumentaron las horas dcdicadas a actividades intensas durante dias festivos, pero en menor proporeion que los vatoncs. Por lo tanto, las niñas, independientemente de su estado nutricional, destinaron mayor número de horas a actividades ligeras. tanto en días de semana como en días festivos.

El gasto energético por actividades diarias en días de semana y festivos en escolares varones resultó mayor en los niños normales que en sus pares de talla baja $(1967 \pm 247$ y $1700 \pm 140$ calorías, respectivamente). Sin embargo, no se establecicron diferencias entre los días de semana y los días festivos, y aun cuando los varones de talla baja aumentaron su gasto cnergético en los feriados, éstc siempre resultó menor en ellos que en los nornales. Al expresar el gasto calórico cn unidades de metabolismo basal, éste corespondió a $1.43 \pm 0.88$ y $1,43 \pm 0,49$ metros, tespectivamente).

El gasto cncrgético por actividades diarias para escolares mujercs también resultó mayor en las nifias normales que en sus pares de talla baja, lanio en los días de semana conto en días festivos (1966 $\pm 184 ; 1252 \pm 246$ semana $y$ $2110 \pm 301 ; 1738 \pm[82 \mathrm{cn}$ festivo), cspecia $\rfloor$ mente en estos últimos se cstableció una diferencia significativa a favor de las niñas nornales. Al expresar el gasto calórico en unidades de metabulismo basal, los valores cortespondieron a 1,42 $\pm 0,84$ para las niñas normales y $1,11 \pm$ 0,89 in para las de talla baja.

En el gasto energético por actividades discrecionales o recreacionales cronometradas, las ni- 
Tabla 3

Distribución del tiempo (h) scgún lipo de actividad en mujeres

Día de senrana y festivo $(\bar{x} \neq D E)$

\begin{tabular}{|c|c|c|c|c|c|c|}
\hline Grupo & $n$ & Sueño & Telerisjon & $\underset{\text { Ligera }}{\text { A ctivid a des }}$ & Moderada & Intensa \\
\hline \multicolumn{7}{|l|}{ Semana } \\
\hline $\begin{array}{l}\text { Normales } \\
\text { Talla baja }\end{array}$ & $\begin{array}{l}17 \\
11\end{array}$ & $\begin{array}{l}11.9 \pm 1.2 \\
12.2 \pm 1.6\end{array}$ & $\begin{array}{l}2.4 \pm 10,8^{*} \\
2.9 \pm 1,1^{*}\end{array}$ & $\begin{array}{l}7.4 \pm 0.4 \\
7,1 \pm 0.3\end{array}$ & $\begin{array}{l}0.4 \pm 0.4 \\
0.4 \pm 0.4\end{array}$ & $\begin{array}{l}1,9 \pm 0,3 \\
1,4 \pm 0,3\end{array}$ \\
\hline \multicolumn{7}{|l|}{ Festivo } \\
\hline $\begin{array}{l}\text { Normales } \\
\text { Talla baja }\end{array}$ & $\begin{array}{l}17 \\
11\end{array}$ & $\begin{array}{l}12,4 \pm 1,5 \\
12,7 \pm 1,2\end{array}$ & $\begin{array}{l}3.4 \pm 1.0)^{*} \\
4.4 \pm 0.8^{*}\end{array}$ & $\begin{array}{l}4,8 \pm 0,6 \\
3,8 \pm 0,6\end{array}$ & $\begin{array}{l}0.6 \pm 0.6 \\
0.5 \pm 0.6\end{array}$ & $\begin{array}{l}2,8 \pm 0,3 \\
2,4 \pm 0,2\end{array}$ \\
\hline
\end{tabular}

${ }^{*} p<0,0001$

ñas notmales mostraron un comportamiento similar a sus pares de talla baja $(2,8 \pm 1,4$ y 2.0 $\pm 0,6 \mathrm{Kcal} / \mathrm{min}$ ). En los varones normales el gasto energético fue significativamente mayor que cn sus pares de talla baja $(4,2 \pm 1,6$ y $2,9 \pm$ $1,3 \mathrm{Kcal} / \mathrm{min}$ ) y que en ambos grupos de mujeres.

En la clasificación de actividades por cronometrado, los varones normales realizaron actividades mínimas o de mantención en una proporción significativamente menor que los de talla baja y que ambos grupos de niñas $(41.4 \%$, $63,9 \%, 70,4 \%$ y $91.1 \%$ respectivamentc). Por cl contrario, en los varones normales la proporción de actividad física intensa fue significativamente mayor que los otros tres grupos $(58,6 \%$ ante $36,1 \%$ en varones de talla baja, $29.6 \%$ niñas normales y $8,9 \%$ en niñas de talla baja).

Los promedios en kilocalorías, proteínas. If pidos e hidralos de carbono, vitaminas y minerales de la ingesta alimentaria (tabla 4) resultaron mayores, aunque las difcrencias no fueron significativas, en los varones y mujeres normales que en sus pares de talla baja. Al conparar estas cifras con recomendaciones de aporte nutritivo para estas edades, el déficit más importante correspondió al calcio, siendo éste de $53 \%$ para el total de la muestra. Las ingestas más bajas de este nutriente se encontraron entre Jos niños de talla baja ( $42 \%$ varones y $47 \%$ mujeres). Otro nutriente deficitario para el total de la muesrra fue la niacina $(66 \%$ de las observaciones). La ingesta de los otros nutrientes analizados resultaron similares a las recomendaciones de aporte para este grupo de edad.

\section{Comentario}

El método cmpleado en el estudio permitió describir estilos de vida en etapa temprana, aun cuando se ha señalado que las principales fuentes de error del mismo son la falta de acuciosidad y veracidad de los registros y la imposibilidad de reproducir fielmente las diferentes actividadcs habituales at estimar el costo energético de las mismas. Es posible reducir cstas limitaciones de la estimación indirecta del gasto energético mediante el manejo de cada técnica por un solo observador, adiestramiento riguroso y empleo de puntos de referencias previamente establecidos en cada prueba.

Los escolares evaluados presentaron caracrerísticas sedentarias, con diforencias de actividad según sexo y estado nutricional concordantes con oras descripciones ${ }^{2-4}$.

El menor compromiso con actividad física intensa y la elevada proporción de tiempo empleado en ver televisión permilirían presumir mayor riesgo en la salud futura de muchos niños. Si bien la televisión está dentro de las actividades ligeras, parece interesante destacar el tiempo destinado a ella por los escolares ante sus implicaciones en el desartollo físico e intelectual del niño. Estílos de vida sedentaria, con escasa actividad física y exceso de televisión promueven la obesidad ${ }^{19}$. Estos resultados deberían ser motivo de alerta, pues la obesidad infantil se está constituyendo en un problema cada vez mayor entre personas de menores rccursos cconómicos, más aún si entre ellos se registran los mayores délicit de crecimiento en 
Tabla 4

Ingesta promedio diaria de calorias y nutrieutes de escolares normales y con talla baja

\begin{tabular}{|c|c|c|c|c|}
\hline & \multicolumn{2}{|c|}{ Varones } & \multicolumn{2}{|c|}{ Mujeres } \\
\hline & Normales & Talla haja & Normales & Talla baja \\
\hline Caloríns (Keal) & $2159 \pm \$ 23$ & $2093 \pm 610$ & $2216 \pm 596$ & $1907 \pm 682$ \\
\hline Poteínas (g) & $60,2 \pm 2.3$ & $59.1 \pm 17$ & $65.7 \pm 21$ & $54.6 \pm 23$ \\
\hline Lipidos (g) & $63,1 \pm 26,9$ & $55,2 \pm 26,4$ & $61.9 \pm 27.9$ & $52,4 \pm 22,7$ \\
\hline H. de C. (g) & $348,6 \pm 113,6$ & $.341,6 \pm 99,7$ & $350,6 \pm 78,1$ & $308,1 \pm 102$ \\
\hline Calcio (ing) & $449 \pm 216$ & $335 \pm 290$ & $512 \pm 250$ & $379 \pm 179$ \\
\hline Hierro (mg) & $14.9 \pm 5.4$ & $14 \pm 5.3$ & $16 \pm 4,9$ & $14 \pm 5.1$ \\
\hline Tisonina (nng) & $1,45 \pm 0.5$ & $1,46 \pm 0,5$ & $1.58 \pm 0.4$ & $1,34 \pm 0.5$ \\
\hline Ribollavinil (mg) & $1.4 \pm 0.7$ & $1,3 \pm 0.6$ & $1,3 \quad \pm 0,5$ & $1,2 \pm 0.7$ \\
\hline Niacina (mg) & $8,2 \pm 3,2$ & $9.0 \pm 3.1$ & $9,0 \quad \pm 3.7$ & $8.0 \pm 3.5$ \\
\hline Vitamina C (ung) & $55.7 \pm 74.7$ & $54.6 \pm 64.1$ & $67.2 \pm 76.2$ & $67.7 \pm 76$ \\
\hline
\end{tabular}

H. de C.: hidratos de carbono

talla ${ }^{20-22}$. Se ha demostrado que existe asociación entre el número de horas destinadas a la telcvisión, la prevalencìa de obesiobad ${ }^{23}, 24, y$ cambios significativos en los hábitos de alimentación, patrones de sueño, rendimiento académico y modificaciones de la actividad fí$\operatorname{sica}{ }^{25}$.

El gasto energélico por actividades diarias calculado en esta expciiencia concuerda con lo descrito en otra ${ }^{26}$ en niños normales de ambos sexos, donde los varones de cualquiera edad eran más activos que las niñas, la actividad física realizada por niños y niñas era limitada , las clases de educación física aportaban los ejercicios de mayor intensidad en su rutina habitual y había tendencias a Ia obesidad.

Con métodos de registro de frecuencia cardíaca se ha encontrado, en niños y niñas de edad escolar, que cl gasto cnergético es significativamente menor en niños con relardo de crecimiento que en controles ${ }^{27}$, resultados similares a los mostrados aquí.

La menor participación de los niños con talla baja en actividades intensas, traduce probahlemente algún tipo de limitación funcional, o bien puede formar parte, junto con al menor crecimiento longitudinal, de las consecuencias de la acción de múltiples factores ambientales.

En general, existe poca información en cuanto a patrones de actividad física en niños del grupo de edad incluido en este estudio. Los trabajos existentes han estado orientados, más que a caracterizar este patrón, a medir gasto ener. gético. La ubilización del registro de frecuencia cardíaca durante períodos prolongados permite hacer esta estimación, si se dispone para cada niño de una curva de calibración de consumo de oxígeno por frecuencia cardíaca, realizada con cargas submáximas de trabajo. La frecuencia cardíaca, de por sí, sin cmbargo, no puede traducirse a niveles de actividad física, ya que a una misma frecuencia, por ejemplo, pueden haber niños realizando un trabajo de tipo moderado $y$ otros un trabajo de lipo incenso o muy intenso, dependiendo de su grado de entrenamiento, de su peso, tamaño y composición corporal, que condicionan la respuesta fisiológica de los niños al ejercicio físico ${ }^{28}$. Un estudio sobre la respuesta fisiológica al ejercicio en escolares, estimado por consumo máximo de $\mathrm{O}_{2}$, demostró que la capacidad de trabajo que realizan los niños a esta edad estaba de acuerdo a su peso y tamaño corporal, lo que condicionaba de alguna manera que los niños con retraso estatural también desarrollaban una menor capaciclad de trabajo físico ${ }^{29}$. En consecuencia, es de interés el aporte de estudios que caractericen a grupos de niños no sólo en término de estimaciones del gasto energético, sino también en basc a patrones de actividad física. El hecho de que tanto el sistema de registro de actividades como cl de observación directa y cronometrado coincidan en señalas la menor dedicación de los niños con talla baja a actividades físicas intensas, sugiere la validez de estos instrumentos para caracterizar patrones de actividad física. De ambos métodos, el registro de acti- 
vidades es el que ofrece mayores posibilidades para su aplicación a grupos más extenșos de niños.

E1 uso de métodos rápidos para caracterizar la actividad física habitual se justifica para hacer intervenciones preventivas, como realización de programas de actividad física en los escolares, que compensen la tendencia al sedentarismo a esa edad y propendan a mejorar la calidad de vida en los adultos. Ellos inclujrían la revisión de los contenidos y métodos empleados en la asignatura de educación física. el diseño de estrategias, para incorporar actividades físicas más exigentes en la vida diaria de niños y adultos, como asimismo crear espacios y medios adecuados para sustentarlos y promoverlos.

\section{Resumen}

En 70 niños y niñas escolares de 7 a 9 años de edad, normales y con talla baja (T/E $\pm 1 \mathrm{DE}$ $y<-1$ DE), se evaluó la actividad física habiutal mediante entrevistas estructuradas y por métudo factorial o de tiempo movimiento. La mayoría de las actividades de estos escolares eran sedentarias. El tiempo dedicado a lroras de sueño o en cama, en dias de la semana y festivos fue muy alto, Ios promedios (talla normal y baja) en los varones eran de $11,5 \pm 1,1$ a $12 \pm$ 1,4 horas y en las niñas $11,9 \pm 1,2$ y de $12,2 \pm$ 1,6 respectivamente. El tiempo destinado a ver televisión era, en varones y niñas. promedio, tres horas diarias, aumentando a cuatro horas en los días ficstivos. Los varones normales destinaron $58,6 \%$ de su tiempo a actividades físicas intensas, porcentaje significativamente mayor que sus pares de talla baja y los dos grupos de niñas ( $\mathrm{p}<0,05$ ). Un comportamiento similar se observó en las niñas normales frente a sus pares de talla baja, pero esta diferencia no alcanzó significación. Así, pues, los escolares de talla baja se involucraron menos en actividades intensas que los normales, y que el ticmpo destinado a ver televisión y la escasa actividad física desarrollada por estos niños, refleja un estilo de vida sedentario por parte de este grupo de edades.

(Palabras clave: actividades diarias, cjercicio, metabolismo energético, consumo, niños escolares.)

\section{Referencias}

1. De Andraca I, Castillo M. Cortés F: Factores de ricsgo para talla bajo en escolares de nivel socioeconómicn bajo. Rev Chil Pediatr 1994: 65: 303-310.

2. Martorell R. Mendoza F. Castillo R: Poverty and stalure in children. In: Linear growth retardation in less developed countries. Ed. J. C. Waterlow. Nestlé Nutrition Worshops Series, Raven Press 1988: 57.74.

3. Nuturowich D. Olivares M. homovich R: Estado nutricional de escolares según sexo y edad, Región Metropolitana, Chije. Rev Med Chil 1990: I18: 916-924.

4. Segunda Reunión de Evaluación Conjunta de la Cooperación Técrica de ta OPS/OMS en Chile: Situación y atención de la saltad en Chile. Santiago, Ministerio de Salud, julio 1993.

5. Viteri FE, Tarin $B$ : Nutrition, physical activity and growih. In: Ritzen M, Peria A, Hale K, Larson A, Zetterberg A, Zeherstrom R. Eds. The biology of normal growth. New York: Raven Press 1981: 265-273.

6. Organización Mustial de la Salard (OMS): Dieta, nutrición y la prevencion de enfermedades crónicas. Ginebra: OMS, 1990 (Serie de informes tecnicos; 797).

7. Washoum RA, Montore HJ: The assesment of physical acrivity by questionnaire. Am J Epideniol 1986: 123: 563-576

8. Sntith GD. Morris JN: Assesment of physical activity and pluysical fitness, in population surveys. J Epidemiol Comm Health 1992; 46: 89-91.

9. Thimothy GL. Roche AF. Martorell R: Anthropometric standarization reference inanual. Abridged edition 1991.

10. OMS: Medición del cambio del estado nutnicional. Ginebra: OMS 1983

11. MiLaren DS. Read WC: Classification of nutrition status in early clildhood. Lances [972; 2: 146-148.

12. Afvarez $M L$. Mezos $S$ Nantrich $D$ : Escala para mediciones del nivel socioeconótnico en el arrea de la salud. Rev Med Chil 1985; 113: 243-249.

13. Astrund P: Tratado de fisiologia del trabajo físico. Editorial Médica Panamericana 1985

14. Schollter DA, Racelte $S B$ : A review of field techniques for the assesment of energy expenditure. J Nutr 1990; 120: 1492-1495.

15. Necesidutes de eneryía y de proterinas: informe do una Reunión Consultiva Conjunta FAO/OMS/UNU de Expertos. Ginebm: OMS, 1985 (Serie de Informes Técnicos): 724

16. Nowris T: Encuestas alimentarias. Su técnica e interpretación, FAO, Estudios de Nutricion $\mathrm{N}^{\circ} 4$. Wachiogton D.C.

17. Duncen $D B$ : Núltiple range and multiple $F$ test. Biometrics 1955; 11: 1-42.

18. Snedecor GW and Cohran WG: Statistical methods. The Jowa State Liniversity Press, AMES, lowa 1967.

19. Antedor $M$ : Adiposity and growth relationship of stature at 14 years with relatire body weight at different ages. E J Clin Nutr 1992; 46: 213-216.

20. Amigo H. Bustos P: Factores condicionantes de la estatura en escolares de comunas pobres de Santiago. Rev Chil Nutr 1992; 20: 167-173,

21. Bustos $P$. Amigo $H$ : Situación nutricional rurat: un esiudio en escolares de zona de alta vulnerabilidad. Rev Chil Pediatr 1993; 64: 392-396. 
22. Butews R, Giadrosiot V, Biolley E, et ol: Estado nutricional y adecuación estatural de escolares en ctapa puheral de diferentes regiones de Chile. Rev Chil Nulr 1992: 20:212-218

23. Dietr. iVH. Gormaker SL: Do we fatten our childnen at the television set? Obesity and television vieweing in children and adolescents. Pediatrics 1985; 75: 807-812

24. Evtein LH. Vatoski A, Varo $L$ et al: Conparative effects of decreasing sedentary behavior and increasing activity on weiglat change in obeje cbildren. Hoalth Psychol I995; 14: 109.115

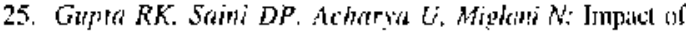
television on thildren. Indian J Pediatr $(4) 4: 60: 153-159$.

26. Riddoch C. Sauge JM. Murphy N. Cran GW. Borehtu C: Long tern health implicalions of jitness and physical activity patterns, Arch Dis Child 1991: 66: $1426-1433$.

27. Spurr GB. Reind $J C$ : Patterns of daily energy expenditure in normal and marsinally undemourished ichool-aged Colombian Children. Eur J Clin Nutr 1988; 42: 819-843.

28. Kamire MA: Repercusiones de los antecodentes mutrcionales y socioeconúmicos sobre el gasto energético diario en niños escolarcs. Tcsis. Universidad Francisco Martoquín, Facultad de Medicina INCAP, Guareinala C.A. 1992.

29. Baburonde C: Diela. composicion corporal y respuesta fisiológica al ejercicio en escolares. Tesis pafa optar al Grado de Migíster en Nutrición Humana. INTA. 1992.

\section{AVISO A LOS AUTORES}

Con el objeto de acelerar la edición de los trabajos se solicita encarecidamente a los autores que disponen de computador con procesadores de texto IBM compatibles, enviar -en lo posible junto con las dos copias impresas de su material-, otra en disco flexible (tamaño 3,5" o 5,25", de doble o alta densidad) del mismo texto. El disco será devuelto de inmediato por correo certificado o por mano al autor corresponsal. 